Huseyin Agah Terzi*, Ozlem Aydemir, Engin Karakece, Huseyin Hatipoglu, Mehmet Olmez, Mehmet Koroglu and Mustafa Altindis

\title{
Investigation of the rapid immunochromatographic test performance in the diagnosis of syphilis; comparison of four serological methods
}

https://doi.org/10.1515/labmed-2019-0012

Received January 22, 2019; accepted June 25, 2020; published online August 6, 2020

\section{Abstract}

Objectives: To test the performance of the newly available rapid test for syphilis, we compared it with Treponema pallidum hemagglutination assay (TPHA). Additionally, we investigated the performance of rapid plasma reagin (RPR) and chemiluminescence microparticle immunoassays (CMIA) at our laboratory using TPHA as a gold standard.

Methods: The serum samples of 595 patients with the prediagnosis of syphilis were studied by four serological methods. The sensitivity, specificity, and predictive values of RPR, CMIA, and syphilis rapid test were assessed by utilizing TPHA as a gold standard for the diagnosis of syphilis.

Results: Of the patients, 6.2\% (37/595) had positive RPR, 5.5\% (33/595) had positive CMIA, 5.5\% (33/595) had a positive rapid immunochromatographic method and 5\% (30/595) had positive TPHA. When TPHA results were taken as the reference, the sensitivity of the rapid test for syphilis was $100 \%$, the specificity was $99.5 \%$, PPV was $90.9 \%$, and NPV was $100.0 \%$. Conclusions: It was observed that the rapid test for syphilis used in the study was quite successful, its cost was appropriate, and the test was very fast and easy to apply. At the same time, the agreement between syphilis rapid test and TPHA was found to be excellent.

Keywords: CMIA; rapid immunochromatographic test; RPR; syphilis serodiagnosis; TPHA.

*Corresponding author: Dr. Huseyin Agah Terzi, Department of Microbiology, Sakarya University, Research/Training Hospital, Sakarya, Turkey, Phone: +90 536 4628654, Fax: +90 26427591 92, E-mail: agah.terzi@yahoo.com Ozlem Aydemir and Engin Karakece, Department of Microbiology, Sakarya University, Research/Training Hospital, Sakarya, Turkey Huseyin Hatipoglu, Mehmet Olmez, Mehmet Koroglu and Mustafa Altindis, Department of Microbiology, School of Medicine, Sakarya University, Sakarya, Turkey

\section{Introduction}

Syphilis represents a sexually transmitted disease (STD) that is caused by the spirochete Treponema pallidum which can be spread by sexual contact, by blood transfusion, and direct contact with lesions during primary and secondary periods [1]. In accordance with the World Health Organization (WHO) reports, about 17.7 million adults (15-49 years) around the world had syphilis in 2012, with estimated 5.6 million new cases each year [2]. Although the prevalence of syphilis varies widely between regions or countries, the highest prevalence rate is in Africa. More than $60 \%$ of new cases are diagnosed in underdeveloped countries [2]. Furthermore, it is estimated that more than $60 \%$ of cases of maternal syphilis worldwide are observed in Africa [3]. As to developed countries, especially in HIV-positive individuals, the incidence has been reported to have increased [1].

Serological methods are used in the diagnosis of syphilis due to the inability to produce the agent in culture. Non-treponemal tests, among the serological methods, used in the laboratory diagnosis of syphilis determine nonspecific antibodies against cardiolipins, while treponemal tests aim to identify specific antibodies that are formed against $T$. pallidum antigens. Venereal disease research laboratory (VDRL) and rapid plasma reagin (RPR) represent non-treponemal tests that are based on the reaction of cardiolipin with nonspecific antibodies generated in response to syphilitic infection [4]. However, these tests have low sensitivity and specificity depending on the pregnancy, autoimmune disorders, other infections and the periods of syphilis disease [5]. Thus, treponemal-specific tests such as enzyme immunoassay (EIA), T. pallidum hemagglutination assay (TPHA), microhemagglutination, fluorescent treponemal antibody absorption test (FTA-ABS), chemiluminescence microparticle immunoassays (CMIA), and the enzyme-linked immunosorbent assay (ELISA) detecting IgG antibodies to antigenic components of T. pallidum are utilized primarily for the purpose of confirming the diagnosis of syphilis in patients with a reactive nontreponemal test $[6,7]$. 
Nowadays, non-treponemal tests are used for screening in the conventional diagnostic algorithm of Syphilis, and positive results are verified by treponemal tests [8]. Treponemal tests are used for screening purposes in the reverse algorithm started to be used in the last decade or in the new diagnostic algorithm of Syphilis, and positive results are verified by a different treponemal test [9]. Treponemal tests are high specificity tests used for verification in the conventional algorithm and for both screening and verification purposes in the inverse/new algorithm. Their disadvantageous aspects are that they are not useful in the follow-up of treatment because they remain positive for years including the patients who have been treated. On the other hand, non-treponemal tests can be used for both screening and treatment follow-up [9].

In the present study, it was aimed to test the performance of the newly available rapid test for syphilis, we compared it with TPHA. Additionally, we investigated the performance of RPR and CMIA at our laboratory using TPHA as a gold standard.

\section{Materials and methods}

\section{Study group}

The serum samples of 595 patients with suspected syphilis that were sent to our laboratory in 2017-2018 (2-year period) were studied by four serological methods. Blood samples were collected from every participant and centrifuged until serum separation, and the actual laboratory tests were conducted. RPR, CMIA, syphilis rapid test, and TPHA were performed, and results were obtained in accordance with the kit's manufacturer's instructions. Characteristics of the methods in this study have shown in Table 1.

\section{Rapid plasma reagin (RPR) test}

Immuntrep RPR (Omega Diagnostics, UK) kit was used for this test. This test is a non-treponemal test for the detection of reagin/cardiolipin antibodies in human serum or plasma by flocculation. The principle of the test is based on the determination of aggregation in samples containing the antibody against the reagins at the end of the comparison of the patient sample and a reagent containing cholesterol/cardiolipin/lecithin. It takes $8 \mathrm{~min}$ to complete the test. The titrations of positive samples were obtained by serial dilutions at the ratios of $1 / 2$ and its multiples to give semiquantitative results. The highest dilution ratio with positivity was reported in the final report.

\section{Chemiluminescence microparticle immunoassay (CMIA)}

Architect Syphilis TP (Abbott Japan Co, Japan) kit was used for this test. It is a treponemal test that detects specific antibodies that are formed against recombinant T. pallidum antigens in human serum or plasma with the help of chemiluminescence reaction. In the evaluation of the test results, the signal to cut-off value $(\mathrm{s} / \mathrm{co})$ ratio calculated by dividing the measurement values of the study samples and control serums by the cut-off value was taken as a basis. This ratio was considered to be negative between 0 and 1 , and positive when higher than 1.0. It takes 26 min to complete the test. In the study, the results of the $\mathrm{s} / \mathrm{co}$ values determined in the CMIA positive samples and the results of the TPHA and RPR tests were compared.

\section{Rapid immunochromatographic assay}

The syphilis rapid test (Hangzhou Biotest Biotech, People's Republic of China) was used for this test. This is a treponemal test that detects IgG and IgM antibodies that are formed against $T$. pallidum by the immunochromatographic method. $75 \mu \mathrm{L}$ of serum sample was used in this test, which could be studied using serum or plasma. The evaluation was made after $15 \mathrm{~min}$.

Table 1: Characteristics of four serological methods in this study.

\begin{tabular}{|c|c|c|c|c|}
\hline Characteristics & RPR & Syphilis rapid test & Architect syphilis & TPHA \\
\hline Method & Agglutination & Immunochromatographic & $\begin{array}{l}\text { Chemiluminescent microparticle immunoassay } \\
\text { (CMIA) }\end{array}$ & Hemagglutination \\
\hline Assay duration & $8 \mathrm{~min}$ & $15 \mathrm{~min}$ & $26 \mathrm{~min}$ & $60 \mathrm{~min}$ \\
\hline Specimen type & Serum & Serum or heparinized plasma & Serum & Serum \\
\hline Sample volume & $50 \mu \mathrm{L}$ & $75 \mu \mathrm{L}$ & $150 \mu \mathrm{L}$ & $10 \mu \mathrm{L}$ \\
\hline Dilutions & Manuel dilution & - & - & Manuel dilution \\
\hline Device & $\begin{array}{l}\text { Omega } \\
\text { Diagnostics }\end{array}$ & Hangzhou Biotest & Architect I 2000 & Omega Diagnostics \\
\hline
\end{tabular}




\section{T. pallidum hemagglutination assay (TPHA)}

A commercial TPHA (Omega Diagnostics, UK) kit was used for this test. TPHA is a treponemal test based on the comparison of patient serums with sensitized erythrocytes in U-bottom microplate wells. Agglutination of sensitized erythrocytes is observed in the patient's serum in the presence of specific $T$. pallidum antibody. The observation of agglutination was evaluated as positive in the $1 / 80$ dilution of the patient serums, and the positive samples were re-studied in further dilutions, and the final titer was reported in the final report.

\section{Statistical analysis}

Number and percentage values were used to define the data. The sensitivity, specificity, and predictive values of RPR, CMIA, and syphilis rapid test in the present study were assessed by utilizing TPHA as a gold standard for the diagnosis of syphilis. The kappa value was calculated to assess the agreement between RPR, CMIA, syphilis rapid test, and TPHA. The kappa coefficient was interpreted as follows: 0.01-0.20, slight agreement; 0.21-0.40, fair agreement; 0.41-0.60, moderate agreement; 0.61-0.80, substantial agreement; 0.81-0.99, excellent/almost perfect agreement. SPSS Statistics 21.0 program (IBM Corp, Armonk, NY, USA) was used in the analyses.

\section{Results}

Of the patients, 6.2\% (37/595) had positive RPR, 5.5\% (33/ 595) had positive CMIA, 5.5\% (33/595) had a positive rapid immunochromatographic method and 5\% (30/595) had positive TPHA. The number of patients who were positive in four methods at the same time was 28 (4.7\%).

When TPHA was taken as the reference method, RPR in seven samples, CMIA in three samples, syphilis rapid test in three samples were found to be false positive. In the syphilis rapid test with RPR, false negative results were not observed, while in the CMIA, false negative results were obtained in two samples. It was observed that the s/co values detected in three samples identified as false positive by the CMIA method were below 12 .

The sensitivity of the rapid immunochromatographic test for syphilis was $100 \%$, the specificity was $99.5 \%$, positive predictive value (PPV) was $90.9 \%$, and negative predictive value (NPV) was $100.0 \%$. The agreement between the TPHA and rapid immunochromatographic test
Table 2: Comparison of Treponema pallidum hemagglutination assay (TPHA) and syphilis rapid test results.

\begin{tabular}{llrrr}
\hline & & & TPHA \\
\cline { 3 - 5 } & & Negative & Positive & Total \\
\hline Syphilis rapid test & Negative & 562 & 0 & 562 \\
& Positive & 3 & 30 & 33 \\
& Total & 565 & 30 & 595 \\
\hline
\end{tabular}

Sensitivity of syphilis rapid test ( 30 of 30 samples), $100 \%$; specificity of syphilis rapid test (562 of 565 samples), $99.5 \%$; agreement $(30+562=592$, of 595 samples), $99.4 \%$; positive predictive value (30/33) 90.9\%; and negative predictive value (562/562) 100\%.

was found to be nearly perfect with a kappa value of 0.99 (Table 2).

When TPHA results were taken as the reference, according to our study, the sensitivity of the RPR test method in the patient group was $100 \%$, the specificity was $98.8 \%$; the PPV was $81.1 \%$ and the NPV was $100.0 \%$ in the diagnosis of syphilis. The agreement between the TPHA and RPR tests was found to be nearly perfect with a kappa value of 0.93 (Table 3).

According to the TPHA results, the sensitivity of the CMIA test was $93.3 \%$, the specificity was $99.1 \%$, PPV was $84.8 \%$, and NPV was $99.6 \%$. The agreement between the TPHA and CMIA tests was found to be nearly perfect with a kappa value of 0.98 (Table 4).

\section{Discussion}

The tests and their order to be selected for the screening and confirmation in the diagnosis of syphilis are important for accurate diagnosis. The conventional algorithm gives more reliable results in populations with high prevalence. Furthermore, the activity of the infection can be determined by this algorithm [10]. The sensitivity and specificity in the conventional algorithm are low, and the false

Table 3: Comparison of TPHA and rapid plasma reagin (RPR) test results.

\begin{tabular}{llrrr}
\hline & & & TPHA \\
\cline { 3 - 5 } & & Negative & Positive & Total \\
\hline RPR & Negative & 558 & 0 & 558 \\
& Positive & 7 & 30 & 37 \\
& Total & 565 & 30 & 595 \\
\hline
\end{tabular}

Sensitivity of RPR ( 30 of 30 samples), $100 \%$; specificity of RPR ( 558 of 595 samples), $98.8 \%$; agreement ( $30+558=588$, of 595 samples), 93.7\%; positive predictive value (30/37) $81.1 \%$; and negative predictive value (558/558) $100 \%$. 
Table 4: Comparison of TPHA and chemiluminescence microparticle immunoassays (CMIA) test results.

\begin{tabular}{llrrr}
\hline & & & TPHA \\
\cline { 3 - 5 } & & Negative & Positive & Total \\
\hline \multirow{2}{*}{ CMIA } & Negative & 560 & 2 & 562 \\
& Positive & 5 & 28 & 33 \\
& Total & 565 & 30 & 595 \\
\hline
\end{tabular}

Sensitivity of CMIA ( 28 of 30 samples), $93.3 \%$; specificity of CMIA ( 560 of 565 samples), $99.1 \%$; agreement ( $28+560=588$, of 595 samples), $98.8 \%$; positive predictive value $(28 / 33) 84.8 \%$; and negative predictive value (560/562) $99.6 \%$.

positivities are determined to be high. Therefore, the results need to be verified [11]. In the inverse/new syphilis diagnosis algorithm, treponemal tests are used as a screening test unlike the conventional algorithm [10]. The results determined as positive are verified by a second and different treponemal test. The inverse algorithm is a costeffective approach in laboratories with a high number of samples, and it gives more reliable results in populations with low syphilis prevalence [12].

Among the non-treponemal tests, the RPR test is often preferred because of its ease of application, rapidity, and cheapness. However, as the cardiolipin antigens used in these tests are not specific, the fact that false positives can be observed in autoimmune patients, pregnant women, and drug addicts is the disadvantage [13, 14]. Among the treponemal tests, the TPHA test is another advantageous test because of its low cost and ease of application. Subjective evaluation and non-automation of the test are its disadvantages [15].

A novel treponemal test group that has started to be used recently and attracted global attention is rapid immunochromatographic tests for syphilis. Among these tests based on the qualitative lateral flow immunochromatographic method, Syphilis Health Check kit is the only test with US Food and Drug Administration (FDA) approval (Trinity Biotech USA, Inc., NY). Differently from conventional laboratory-based syphilis treponemal tests, syphilis rapid tests (RST) are portable to be used in the field, are relatively cheap and do not include complex training [16]. In the studies carried out with Syphilis Health Check (SHC), the sensitivity was found to be $>95 \%$, and the specificity was found to be $>90 \%$ [16-18]. The sensitivity of the rapid immunochromatographic test for syphilis in our study was $100 \%$, the specificity was $99.5 \%$, PPV was $90.9 \%$, and NPV was $100.0 \%$. The agreement between the TPHA and syphilis rapid tests was found to be nearly excellent/perfect agreement with a kappa value of 0.98 (Table 2).
In various studies, the sensitivity and specificity of RPR were comparable to the findings reported in our study [1921]. The RPR specificity in our study was determined to be $98.8 \%$. Similar results were obtained in different studies (96.9 and 100\%) [22, 23]. The difference in the high specificity values may be due to reasons such as commercial kit, subjective evaluation, personnel experience. For example, the reduction of the margin of human error due to the preference of the automated system in the mentioned studies may have increased the specificity. In our study, the RPR positive and TPHA negative results of the $9(1.7 \%)$ patients obtained by the conventional algorithm were evaluated as false positive reactions.

Another treponemal test used in the laboratory diagnosis of syphilis is CMIA. This test is studied using automated chemiluminescence devices. In this way, a large number of serum samples can be studied at the same time, and the results are evaluated objectively [24, 25]. Therefore, the use of CMIA is appropriate for centers with a high number of patients [24]. Studies with similar sensitivity and specificity (90-100\%) were found in the literature [26-30]. However, some studies reported different values from our study [31-33]. This change in the performance of the CMIA test is due to the type of immunodominant syphilis proteins. The PPV and NPV values of the CMIA test were determined as 84.8 and $98.8 \%$, respectively. Similar PPV and NPV values were also reported in various studies [26, $28,32]$.

In our study, the CMIA results of $5(0.8 \%)$ patients evaluated as CMIA positive and TPHA negative were determined to be false positive. Moreover, the presence of a relationship between the TPHA results and CMIA values was also investigated, and CMIA positive cases below $12 \mathrm{~s} /$ co were found to be TPHA negative. Although this result was not verified by another method, which is accepted as a gold standard such as FTA-ABS, it suggests that the CMIA test may give false positive results at low s/co values. Therefore, CMIA results were suggested to be repeated one month later in accordance with the diagnosis algorithm for CMIA positive and TPHA negative samples. There are also studies in the literature indicating that the CMIA test can give false positive results at low s/co values. Young et al. reported that the s/co values of the samples which were stated as false positives were $\leq 5$ [34]. Similarly to our study, Ozbek et al. reported that $\mathrm{s} / \mathrm{co}$ values give false positive results when they are $<12$ [35]. According to the data we obtained, low s/co values have a risk of false positivity. However, in accordance with the guidelines published in recent years, it would be appropriate to use the treponemal tests including the CMIA and the rapid immunochromatographic test for syphilis in screening. In case of a 
positivity was detected in screening, a verification should be performed with treponemal tests such as TPHA and FTA-ABS. Also, RPR can be studied to contribute to the evaluation of the patient in terms of active syphilis and to make a possible treatment follow-up.

Unfortunately, our study had a limited number of syphilitic patients because of the low prevalence of syphilis in our country, so the number of samples was small and could not been classified according to syphilis stage. Furthermore, samples evaluated as TPHA positive were not sent to the reference laboratory for verification by the FTA-ABS test.

In conclusion, it was observed that the rapid immunochromatographic test for syphilis (sensitivity, specificity, and predictive values) used in the study was quite successful, its cost was appropriate, and the test was very fast and easy to apply. It can be used for screening and diagnosis. At the same time, the agreement between syphilis rapid immunochromatographic test and TPHA was found to be excellent.

Research funding: None declared.

Author contributions: All authors have accepted responsibility for the entire content of this manuscript and approved its submission.

Competing interests: Authors state no conflict of interest. Informed consent: Informed consent was obtained from all individuals included in this study.

Ethical approval: Research involving human subjects complied with all relevant national regulations, institutional policies and is in accordance with the tenets of the Helsinki Declaration (as revised in 2013), and has been approved by the authors' Institutional Review Board.

\section{References}

1. Tramont EC. Treponema pallidum (syphilis). In: Mandell GL, Bennett JE, Dolin R, editors. Principles and practice of infectious diseases, 7th ed. New York: Churchill Livingstone; 2010:3035-55 pp.

2. Newman L, Rowley J, Vander Hoorn S, Wijesooriya NS, Unemo M, Low N, et al. Global estimates of the prevalence and incidence of four curable sexually transmitted infections in 2012 based on systematic review and global reporting. PLoS One 2015;10: e0143304.

3. Wijesooriya NS, Rochat RW, Kamb ML, Turlapati P, Temmerman M, Broutet N, et al. Global burden of maternal and congenital syphilis in 2008 and 2012: a health systems modelling study. Lancet Glob Health 2016;4:e525-33.

4. Luger AF. Serological diagnosis of syphilis: current methods. In: Young H, McMillan A, editors. Immunological diagnosis of sexually transmitted diseases. New York: Dekker; 1988:250-9 pp.
5. Fischbach FT. Syphilis detection tests. In: A manual of laboratory \& diagnostic tests, 6th ed. Philadelphia: Lippincott; 2000:581-3 pp.

6. Young H, Moyes A, Seagar L, McMillan A. Novel recombinantantigen enzyme immunoassay for serological diagnosis of syphilis. J Clin Microbiol 1998;36:913-7.

7. Carlsson B, Hanson HS, Wasserman J, Brauner A. Evaluation of the fluorescent treponemal antibody-absorption (FTA-ABS) test specificity. Acta Derm Venereol 1991;71:306-11.

8. Simčič $S$, Potočnik $M$. Serological diagnosis of syphilis: a comparison of different diagnostic methods. Acta

Dermatovenerol Alp Pannonica Adriat 2015;24:17-20.

9. Tong ML, Lin LR, Liu LL, Zhang HL, Huang SJ, Chen YY, et al. Analysis of 3 algorithms for syphilis serodiagnosis and implications for clinical management. Clin Infect Dis 2014;58: 1116-24.

10. Binnicker MJ, Jespersen DJ, Rollins LO. Treponema-specific tests for serodiagnosis of syphilis: comparative evaluation of seven assays. J Clin Microbiol 2011;49:1313-7.

11. Centers for Disease Control and Prevention (CDC). Discordant results from reverse sequence syphilis screening - five Laboratories, United States, 2006-2010. MMWR 2011;60: 133-7.

12. Janier M, Hegyi V, Dupin N, Unemo M, Tiplica GS, Potočnik M, et al.. 2014 European guideline on the management of syphilis. J Eur Acad Dermatol Venereol 2014;28:1581-93.

13. Aktaş G. Serological diagnosis of syphilis. Türk Mikrobiyol Cem Derg 2005;35:73-9.

14. Goh BT, van Voorst Vader PC. European guideline for the management of syphilis. Int J STD AIDS 2001;12:14-26.

15. Larsen SA, Steiner BM, Rudolph AH. Laboratory diagnosis and interpretation of tests for syphilis. Clin Microbiol Rev 1995;8: 1-21.

16. Pereira LE, McCormick J, Dorji T, Kang J, Sun Y, Shukla M, et al. Laboratory evaluation ofna commercially available rapid syphilis test. J Clin Microbiol 2018;56:e00832-18.

17. Matthias J, Dwiggins P, Totten Y, Blackmore C, Wilson C, Peterman TA. Notes from the field: evaluation of the sensitivity and specificity of a commercially available rapid syphilis test - Escambia County, Florida. MMWR 2016;2016: 1174-5.

18. Nakku-Joloba E, Kiragga A, Mbazira JK, Kambugu F, Jett-Goheen M, Ratanshi RP, et al. Clinical evaluation of 2 point of-care lateral flow tests for the diagnosis of syphilis. Sex Transm Dis 2016;43: 623-5.

19. Dumre SP, Shakya G, Acharya D, Malla S, Adhikari N. Diagnostic dilemma of the single screening test used in the diagnosis of syphilis in Nepal. Nepal Med Coll J 2011;13:238-40.

20. Lee JH, Lim CS, Lee MG, Kim HS. Comparison of an automated rapid plasma reagin (RPR) test with the conventional RPR card test in syphilis testing. BMJ Open 2014;4:e005664.

21. Rakotoarisoa A, Andriamandimbisoa H, Randriamahazo T, Andrianavalona J, Rajaonatahiana D, Rasamindrakotroka A. Performance of SD bioline syphilis 3.0 for the diagnosis of sypilis a UPFR in immunology of CHU-JRA. Int J Curr Microbiol Appl Sci 2017;6:783-8.

22. Paul M, Sen SS. A comparative study of clinically suspected syphilis cases with serological test VDRL/RPR \& Treponema pallidum hemagglutination assay (TPHA) in a tertiary care hospital (Silchar Medical College \& Hospital). J Sci 2016;6: 521-6. 
23. Henning MP, Krüger C, Fletcher L. Syphilis seropositivity in recently admitted and long-term psychiatric inpatients: screening, prevalence and diagnostic profile. S Afr J Pscyh 2012; 18:171-5.

24. Egglestone SI, Turner AJ. Serological diagnosis of syphilis. PHLS syphilis working group. Commun Dis Public Health 2000;3: 158-62.

25. Goh BT, van Voorst Vader PC. European guideline for the management of syphilis. Int J STD AIDS 2001;12:14-26.

26. Saral Y, Dilek AR, Dilek N, Bahçeci I, Ulusan DZ. Serologic diagnosis of syphilis: comparison of different diagnostic methods. Acta Dermatovenerol Croat 2012;20:84-8.

27. Ozolins D, Katkovska S, Bobojeda L, Rancane A. Screening assays to find out late latent syphilis cases-which is the best one?. Internet J Med Update 2009;4:29-35.

28. Woznicová V, Vališová Z. Performance of CAPTIA SelectSyph-G enzyme-linked immunosorbent assay in syphilis testing of a high-risk population: analysis of discordant results. J Clin Microbiol 2007;45:1794-7.

29. Ebel A, Vanneste L, Cardinaels M, Sablon E, Samson I, De Bosschere K, et al. Validation of the INNO-LIA syphilis kit as a confirmatory assay for Treponema pallidum antibodies. J Clin Microbiol 2000;38:215-9.
30. Zrein M, Maure I, Boursier F, Soufflet L. Recombinant antigenbased enzyme immunoassay for screening of Treponema pallidum antibodies in blood bank routine. J Clin Microbiol 1995; 33:525-7.

31. Tsang RSW, Martin IE, Lau A, Sawatzky P. Serological diagnosis of syphilis: comparison of the Trep-Chek IgG enzyme immunoassay with other screening and confirmatory tests. FEMS Immunol Med Microbiol 2007;51:118-24.

32. Rodriguez I, Alvarez EL, Fernandez C, Miranda A. Comparison of a recombinant-antigen enzyme immunoassay with Treponema pallidum hemagglutination test for serological confirmation of syphilis. Mem Inst Oswaldo Cruz 2002;97:347-9.

33. Naidu NK, Bharucha ZS, Sonawane V, Ahmed I. Comparative study of treponemal and non-treponemal test for screening of blood donated at a blood center. Asian J Transfus Sci 2012;6: 32-5.

34. Young H, Pryde J, Duncan L, Dave J. The Architect Syphilis assay for antibodies to Treponema pallidum: an automated screening assay with high sensitivity in primary syphilis. Sex Transm Infect 2009;85:19-23.

35. Ozbek OA, Doğan Y. Evaluation of a syphilis testing algorithm using a treponemal test for screening. Mikrobiyol Bul 2011;45: 93-103. 